\title{
Space-Frequency Equalization for Broadband Single Carrier MIMO Systems
}

\author{
Gayathri Kongara, Desmond P. Taylor and Philippa A. Martin \\ Department of Electrical and Computer Engineering \\ University of Canterbury, Private Bag 4800, Christchurch, New Zealand \\ (gko19@student, taylor@elec, p.martin@elec).canterbury.ac.nz
}

\begin{abstract}
In this paper, a frequency domain (FD) receiver architecture implemented using estimated channel parameters is derived for broadband single carrier modulations. Co-channel and inter-symbol interferences are compensated by a minimum mean squared error based integrated space-frequency-equalizer (SFE) using the estimated parameters. The integrated SFE in the FD consists of coupled FIR structures, that are jointly optimized by maximizing the desired signal to interference plus noise ratio. We develop analytical expressions and present simulation results for the integrated SFE. Simulation results demonstrate that excellent receiver performance is achieved even for channels with large ISI spans. The proposed SC-SFE outperforms previous layered space frequency (LSF) receivers [1] with imperfect channel knowledge. Further, the parallel receiver architecture yields equal diversity gains to all data streams without the error propagation that is common to most LSF schemes.
\end{abstract}

\section{INTRODUCTION}

The effect of multipath propagation increases with increasing data rate for single carrier (SC) broadband wireless access (BWA) systems. This leads to larger inter-symbolinterference (ISI) spans. The achievable capacity of SC-BWA systems depends on their ability to accommodate multiple signal transmissions in the same frequency band, which results in co-channel interference $(\mathrm{CCI})$ when detecting the desired data stream.The effects of CCI and ISI are more pronounced at higher data rates. The optimum receiver for channels impaired by CCI and ISI is the multichannel maximum likelihood sequence estimation equalizer [2]. However, its implementation complexity is infeasible in the presence of CCI. Hence, SC-BWA receivers are required to compensate the effects of fading, CCI, ISI and additive white Gaussian noise (AWGN) at a practical implementation complexity.

In the last decade, the efficacy of frequency-domain equalization (FDE) for single-carrier (SC) modulation in heavily dispersive environments has been identified and is proposed as a solution for future BWA systems [3]. As with orthogonal frequency division multiplexing (OFDM), SC-FDE systems have low complexity equalization but have the added advantages of lower peak to average transmitted power ratio and less sensitivity to phase noise and frequency offset.

Spatial multiplexing (SM) and space-time coding are the two most used multiple-input multiple-output (MIMO) techniques [4], [1], [5] and [6]. Both linear and non-linear receiver architectures have been studied in the framework of SCFDE systems. These have been extended to the Vertical Bell
Laboratories Layered Space-Time (V-BLAST) architectures by introducing FD CCI cancellation across layers or data streams [7], [8], [1], [5]. Successive and parallel interference cancellation (SIC and PIC) schemes have been proposed for SM-SC systems [8], [9], [1]. The SIC scheme of [9] assumes perfect channel state information to implement a FD channel matched filter followed by an MMSE equalizer. Optimal detection ordering and perfect CCI cancellation are assumed in [9]. However, in practice imperfect channel knowledge affects the detection ordering and degrades the performance because of error propagation. Further, not all transmitted data streams fully exploit the available diversity of the MIMO multipath channel.

In [1], FD linear and decision feedback equalizers (DFEs) in conjunction with SIC were proposed for spatially multiplexed SC systems. The receiver operations for SIC in [1] are equivalent to the generalized MIMO DFE for SM-SC systems. Multiple stages involved in the PIC and SIC schemes with a linear equalizer or a DFE at each stage accentuate the effect of error propagation under imperfect channel knowledge [1]. In this paper, we develop an integrated SFE receiver, that equalizes CCI and ISI jointly in the FD without error propagation. Further, with the integrated SFE, similar performance is achieved for all transmitted data streams.

In [10], [7], FD channel estimation algorithms for MIMO SC-FDE systems were proposed. The use of FD orthogonal Chu sequences for training in [10], reduces the effect of CCI during the channel estimation period. It can be shown that channel estimation in the time domain requires computation of fewer parameters than in the FD [11]. Hence, we propose a training-sequence-based, time-domain joint estimation algorithm for the estimation of the receiver parameters used in CCI and ISI suppression. The receiver vector parameters so obtained are transformed to the FD to construct the integrated SFE. Unlike [10], [7], the training sequences are not required to be orthogonal in either the time domain or the FD.

The rest of the paper is organized as follows. In Section II the system and channel models are described. Section III describes the joint estimation algorithm and the derivation of the time domain channel parameters. Section IV derives the FD integrated SFE. In Section V, simulation results are discussed.

Notation: Upper case bold italic font is used to represent FD vectors (e.g., $\mathcal{H}$ ) and lower case bold font is used for 
time domain vectors (e.g., h). A matrix is represented with a bar on top of the corresponding variables (e.g., $\overline{\mathcal{H}}$ ) and $\overline{\mathbf{h}}$. $E[$.$] is used to denote the expected value of a random process,$ and $\|.\|^{2}$ denotes the squared Euclidean norm. The operators $(.)^{T},(.)^{*},(.)^{H}$ and $(.)^{-1}$ are used to represent the transpose, complex conjugate, complex conjugate transpose and inverse operations, respectively.

\section{System Model}

For SC MIMO systems, the spatio-temporal interference due to CCI and ISI can be compensated by means of a spacetime or a space-frequency equalizer (STE or SFE). Some space-frequency processors attempt to suppress CCI and ISI simultaneously, [12], [8], [13] and [7]. Fig. 1 illustrates the proposed integrated SFE implementing a training sequence assisted joint estimation approach. The receiver consists of $M$ parallel branches each corresponding to a transmitted data stream. The frequency transformed received signals from $N \geq M$ receive antennas are fed to all $M$ receiver branches. When detecting data from the $m^{\text {th }}$ transmitter, signals received from all other transmitters are considered to be CCI. The $m^{t h}$ branch performs CCI suppression, diversity combining and ISI equalization in the FD. In Section IV, the analytical model for the integrated SFE is derived.

The filter parameters used in the integrated SFE are computed by a training sequence based joint estimation algorithm that maximizes the desired signal to interference plus noise ratio (SINR) as in [14]. The signal from the $N$ receive antennas during the training period are used by the joint estimation algorithm. This yields two vector parameters, $\mathbf{w}_{m, n}$ and $\mathbf{f}_{m}$ which jointly suppress CCI and model the composite channel in the time domain. This time domain approach to channel estimation requires computation of fewer channel parameters than the FD approaches for data detection [11].

Consider a SC-BWA system with $M$ transmit antennas and $N$ receive antennas communicating over a frequency selective Rayleigh fading MIMO channel. The data is multiplexed into $M$ independent frames of length $N_{s}$ symbols which are simul-

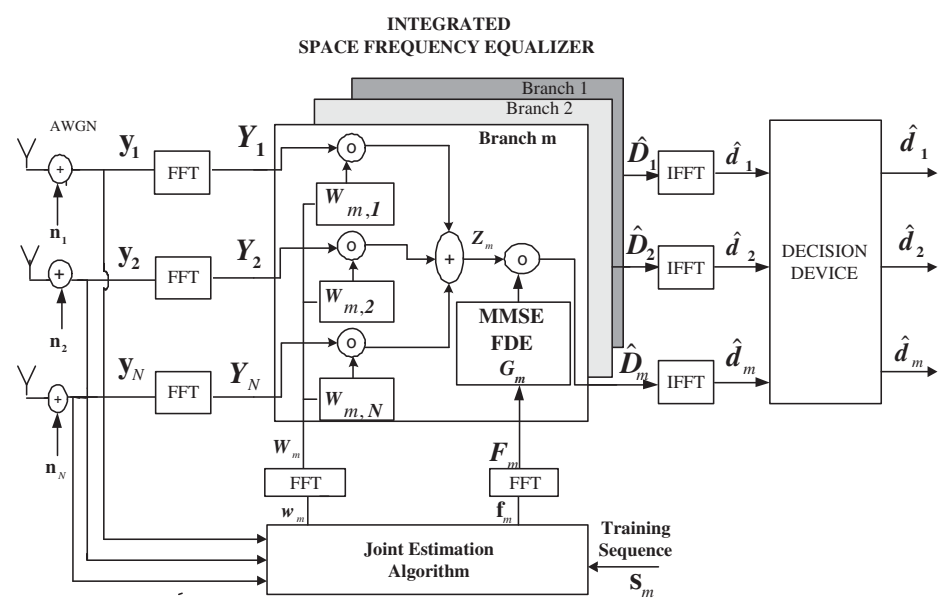

Fig. 1. The proposed integrated SFE receiver.

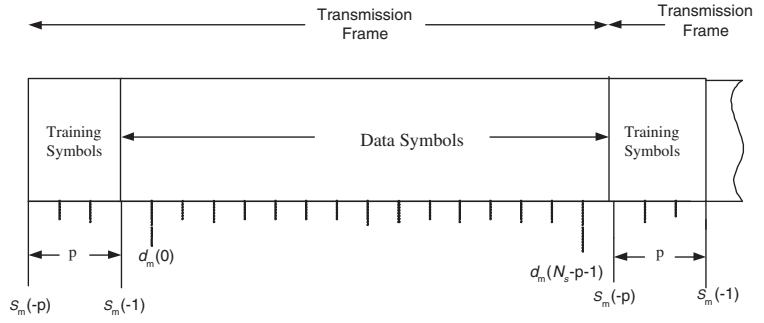

Fig. 2. Transmission frame format

taneously transmitted from $M$ spatially independent antennas. The classical approach to avoiding inter-frame interference due to multipath propagation is the use of a cyclic prefix/postfix (CP) of length at least equal to the maximum expected channel delay spread [3]. Here, we use a periodic pseudo random training sequence of length $p$ as shown in Fig 2. The current transmission frame from the $m^{\text {th }}$ transmit antenna is denoted

$\mathbf{d}_{m}=\left[s_{m}(-p), \ldots, s_{m}(-1), d_{m}(0), \ldots, d_{m}\left(N_{s}-p-1\right)\right]$,

where $d_{m}(j)$ denotes the $j^{\text {th }}$ data symbol in the frame and $s_{m}(j)$ denotes the $(p-j+1)^{t h}$ symbol in the training sequence.

We denote the channel impulse response vector ${ }^{1}$ with $v$ taps corresponding to the path between the $m^{\text {th }}$ transmitter and the $n^{\text {th }}$ receive antenna by

$$
\mathbf{h}_{m, n}=\left[\begin{array}{llll}
h_{m, n}(0) & h_{m, n}(1) & \ldots & h_{m, n}(v-1)
\end{array}\right] .
$$

A convolution channel matrix $\overline{\mathbf{h}}_{m, n}$ of dimension $(L+1) \times$ $(L+v+1)$ is then defined as

$$
\left[\begin{array}{ccccc}
h_{m, n}(0) & \ldots & h_{m, n}(v-1) & \ldots & 0 \\
0 & h_{m, n}(0) & \ldots & \ldots & 0 \\
\vdots & \ddots & \ddots & \ddots & \vdots \\
0 & \ldots & \ldots & \ldots & h_{m, n}(v-1)
\end{array}\right]
$$

We now consider the $m^{\text {th }}$ transmitted signal as the desired signal and the signals from the other $M-1$ transmitters as interference. After discarding the first $p$ training symbols we can write the noisy received signal matrix at the $n^{\text {th }}$ receiver as

$$
\overline{\mathbf{y}}_{n}=\overline{\mathbf{h}}_{m, n} \overline{\mathbf{d}}_{m}+\sum_{\forall i \neq m} \overline{\mathbf{h}}_{i, n} \overline{\mathbf{d}}_{i}+\overline{\mathbf{n}}_{n},
$$

where the $(L+v+1) \times\left(N_{s}\right)$ matrix $\overline{\mathbf{d}}_{m}$ is given by

$$
\left[\begin{array}{cccc}
d_{m}(0) & d_{m}(1) & \ldots & s_{m}(-1) \\
s_{m}(-1) & d_{m}(0) & \ddots & s_{m}(-2) \\
\vdots & \ddots & \ddots & \vdots \\
s_{m}(-L-v) & \ldots & \ldots & s_{m}(-L-v-1)
\end{array}\right]
$$

and $\overline{\mathbf{n}}_{n}$ is an $(L+1) \times\left(N_{s}\right)$ AWGN matrix.

\footnotetext{
${ }^{1}$ We assume that the channel impulse response vector remains static over a frame and varies independently across frames.
} 


\section{Joint Estimation Algorithm}

The FIR filter vector $\mathbf{w}_{m, n}$ suppresses the dispersive $\mathrm{CCI}$ at the $n^{\text {th }}$ receiver and is referred to as the space time processor (STP) response. The vector $\mathbf{f}_{m}$ is the effective channel impulse response vector which includes the filtering delay due to the multipath channel and the STP. Joint optimization of $\mathbf{w}_{m, n}$ and $\mathbf{f}_{m}$ is achieved by maximizing the desired signal to interference plus noise ratio (SINR) through use of Eigen analysis. This process is carried out with the help of the received training matrix, which is corrupted by CCI and ISI. The $(L+v+1) \times(p)$ training matrix is defined as

$$
\overline{\mathbf{s}}_{m}=\left[\begin{array}{cccc}
s_{m}(-p) & s_{m}(-p+1) & \ldots & s_{m}(-1) \\
s_{m}(-1) & s_{m}(-p) & \ddots & s_{m}(-2) \\
\vdots & \ddots & \ddots & \vdots \\
s_{m}(-L-v) & \ldots & \ldots & s_{m}(-L-v-1)
\end{array}\right]
$$

Similar to (4), we can write the received signal during the training period as

$$
\overline{\mathbf{y}}_{n}^{s}=\overline{\mathbf{h}}_{m, n} \overline{\mathbf{s}}_{m}+\sum_{\forall i \neq m} \overline{\mathbf{h}}_{i, n} \overline{\mathbf{s}}_{i}+\overline{\mathbf{n}}_{n}, \quad i \in[1 \ldots M]
$$

The last two terms consist of CCI and noise. This can be written as a single disturbance term denoted as $\overline{\mathbf{e}}_{m, n}$. Then, (7) can be written as

$$
\overline{\mathbf{y}}_{n}^{s}=\overline{\mathbf{h}}_{m, n} \overline{\mathbf{s}}_{m}+\overline{\mathbf{e}}_{m, n}
$$

On stacking $N$ such matrices we have,

$$
\overline{\mathbf{y}}^{s}=\left[\begin{array}{c}
\overline{\mathbf{y}}_{1}^{s} \\
\overline{\mathbf{y}}_{2}^{s} \\
\vdots \\
\overline{\mathbf{y}}_{N}^{s}
\end{array}\right]
$$

The design goal of the vector

$$
\mathbf{w}_{m, n}=\left[\begin{array}{llll}
w_{m, n}(0) & w_{m, n}(1) & \ldots & w_{m, n}(L)
\end{array}\right]
$$

is to suppress the CCI due to the $M-1$ co-channel signals. The overall STP weight vector $\mathbf{w}_{m}$ is obtained by concatenating $N$ vectors corresponding to the $N$ receive antennas giving,

$$
\mathbf{w}_{m}=\left[\begin{array}{llll}
\mathbf{w}_{m, 1} & \mathbf{w}_{m, 2} & \ldots & \mathbf{w}_{m, N}
\end{array}\right] .
$$

The overall output after space-time processing of $\overline{\mathbf{y}}^{s}$ is then written as

$$
\begin{aligned}
\mathbf{z}_{m}^{s} & =\sum_{n=1}^{N}\left(\mathbf{w}_{m, n} \overline{\mathbf{h}}_{m, n} \overline{\mathbf{s}}_{m}+\mathbf{w}_{m, n} \overline{\mathbf{e}}_{m, n}\right) \\
& =\mathbf{w}_{m} \overline{\mathbf{y}}^{s} .
\end{aligned}
$$

The effective channel impulse response vector between the $m^{\text {th }}$ transmitter and the output of the STP is defined as

$$
\mathbf{f}_{m}=\sum_{n=1}^{N} \mathbf{w}_{m, n} \overline{\mathbf{h}}_{m, n},
$$

Likewise, the resulting disturbance is given by

$$
\mathbf{i}_{m}=\sum_{n=1}^{N} \mathbf{w}_{m, n} \overline{\mathbf{e}}_{m, n} .
$$

Substituting (14) and (15) in (12) and equating to the right hand side of (13) we have

$$
\mathbf{z}_{m}^{s}=\mathbf{f}_{m} \overline{\mathbf{s}}_{m}+\mathbf{i}_{m}=\mathbf{w}_{m} \overline{\mathbf{y}}^{s},
$$

where $\mathbf{f}_{m} \overline{\mathbf{s}}_{m}$ corresponds to the desired data stream component. We can now write the objective function (SINR) for detecting the $m^{\text {th }}$ data stream as [14]

$$
\begin{aligned}
J_{m}\left(\mathbf{w}_{m}, \mathbf{f}_{m}\right) & =\frac{\left\|\mathbf{f}_{m} \overline{\mathbf{s}}_{m}\right\|^{2}}{\left\|\mathbf{i}_{m}\right\|^{2}} \\
& =\frac{\left\|\mathbf{f}_{m} \overline{\mathbf{s}}_{m}\right\|^{2}}{\left\|\mathbf{w}_{m} \overline{\mathbf{y}}^{s}-\mathbf{f}_{m} \overline{\mathbf{s}}_{m}\right\|^{2}} .
\end{aligned}
$$

Maximizing $J_{m}$ is equivalent to minimizing its denominator with respect to the vectors $\mathbf{w}_{m}$ and $\mathbf{f}_{m}$. To do this, we first find a general solution for $\mathbf{w}_{m}$ as a function of $\mathbf{f}_{m}$ by minimizing the denominator of $J_{m}$ subject to a unit energy constraint ${ }^{2}$ on $\mathbf{f}_{m}$. Taking the partial derivative of the denominator of (18) with respect to the $j^{t h}$ element of $\mathbf{w}_{m}$ and equating the result to zero we have

$$
\frac{\partial}{\partial\left(\mathbf{w}_{m}\right)_{j}}\left\|\mathbf{w}_{m} \overline{\mathbf{y}}^{s}-\mathbf{f}_{m} \overline{\mathbf{s}}_{m}\right\|^{2}=0, \quad j=1,2, \ldots N(L+1) .
$$

On solving the system of equations resulting from (19) it can be shown that

$$
\mathbf{w}_{m}=\mathbf{f}_{m}\left(\overline{\mathbf{s}}_{m}\left(\overline{\mathbf{y}}^{s}\right)^{H}\right)\left(\overline{\mathbf{y}}^{s}\left(\overline{\mathbf{y}}^{s}\right)^{H}\right)^{-1} .
$$

This solution is then substituted into (18) to obtain the objective function in terms of $\mathbf{f}_{m}$ alone as

$$
J_{m}\left(\mathbf{f}_{m}\right)=\frac{\left\|\mathbf{f}_{m} \overline{\mathbf{s}}_{m}\right\|^{2}}{\left\|\mathbf{f}_{m} \overline{\mathbf{s}}_{m}\left(\overline{\mathbf{y}}^{s}\right)^{H}\left(\overline{\mathbf{y}}^{s}\left(\overline{\mathbf{y}}^{s}\right)^{H}\right)^{-1} \overline{\mathbf{y}}^{s}-\mathbf{f}_{m} \overline{\mathbf{s}}_{m}\right\|^{2}} .
$$

Maximization with respect to $\mathbf{f}_{m}$ is now accomplished by expanding (21) to the form

$$
J_{m}\left(\mathbf{f}_{m}\right)=\frac{\mathbf{f}_{m} \overline{\mathbf{s}}_{m} \overline{\mathbf{s}}_{m}^{H} \mathbf{f}_{m}^{H}}{\mathbf{f}_{m} \overline{\mathbf{s}}_{m}\left(\mathbf{I}-\left(\overline{\mathbf{y}}^{s}\right)^{H}\left(\overline{\mathbf{y}}^{s}\left(\overline{\mathbf{y}}^{s}\right)^{H}\right)^{-1} \overline{\mathbf{y}}^{s}\right) \overline{\mathbf{s}}_{m}^{H} \mathbf{f}_{m}{ }^{H}} .
$$

The optimal $\mathbf{f}_{m}$ is the Eigenvector corresponding to the maximum Eigenvalue of $\left[\overline{\mathbf{s}}_{m}\left(\mathrm{I}-\left(\overline{\mathbf{y}}^{s}\right)^{H}\left(\overline{\mathbf{y}}^{s}\left(\overline{\mathbf{y}}^{s}\right)^{H}\right)^{-1} \overline{\mathbf{y}}^{s}\right) \overline{\mathbf{s}}_{m}^{H}\right]^{-1}\left(\overline{\mathbf{s}}_{m} \overline{\mathbf{s}}_{m}^{H}\right) . \quad$ The optimal $\mathbf{w}_{m}$ is then found by substituting the resulting normalized Eigen vector into (20). It can be shown that the optimal receiver parameters so computed yield the global maximum for the objective function of (18) [14].

\footnotetext{
${ }^{2}$ The constraint $\left\|\mathbf{f}_{m}\right\|^{2}=1$ is used to avoid any degenerate solution resulting from the optimization process.
} 


\section{MMSE SPACE FREQUENCY EQUALIZER}

Space-frequency processing is an effective low complexity equalizer solution for SC-BWA systems. Complexity reduction is achieved based on the discrete convolution theorem which enables the utilization of the fast-Fourier transform (FFT) algorithm. Equalizer complexity is proportional to the logarithm of the FFT block length. Hence, the vector parameters derived in Section III are frequency transformed to construct the integrated SFE. It consists of $M$ parallel branches each corresponding to a transmitter, here we consider the $m^{t h}$ branch. At the $k^{\text {th }}$ frequency tone, the time domain vectors $\mathbf{w}_{m, n}$ and $\mathbf{f}_{m}$ are frequency transformed as

$$
\begin{aligned}
& \mathcal{W}_{m, n}\left(\frac{2 \pi}{N_{s}} k\right)=\frac{1}{N_{s}} \sum_{j=0}^{L} \mathrm{w}_{m, n}(j) e^{-j 2 \frac{\pi}{N_{s}} k j} \\
& \mathcal{F}_{m}\left(\frac{2 \pi}{N_{s}} k\right)=\frac{1}{N_{s}} \sum_{j=0}^{v+L} \mathrm{f}_{m}(j) e^{-j 2 \frac{\pi}{N_{s}} k j} \\
& k=1,2, \ldots . N_{s} .
\end{aligned}
$$

The vector $\mathcal{W}_{m, n}$, performs SFP to mitigate the effects of dispersive CCI and to maximize the desired SINR. The residual CCI and the ISI in the desired data stream are then compensated by an MMSE based FDE constructed based on the effective channel frequency response vector $\mathcal{F}_{m}$. The overall processor thus performs linear equalization in the space and frequency domains. The frequency transformed received signal at the $n^{\text {th }}$ receiver is given by

$$
\mathcal{Y}_{n}=\mathcal{H}_{m, n} \circ \mathcal{D}_{m}+\sum_{\forall i \neq m} \mathcal{H}_{i, n} \circ \mathcal{D}_{i}+\mathcal{N}_{m, n}
$$

where $\circ$ represents the Hadamard product or component-wise multiplication of vectors. Circular convolution of vectors in the time domain is equivalent to their Hadamard product in the FD. The FD vector $\mathcal{D}_{m}$ corresponds to $N_{s}$ FFT samples of the transmission frame. Vectors $\mathcal{H}_{m, n}$ and $\mathcal{N}_{m, n}$ are the channel frequency response and frequency transformed AWGN samples, respectively. The FD received signal is represented as

$$
\mathcal{Y}_{n}=\mathcal{H}_{m, n} \circ \mathcal{D}_{m}+\mathcal{E}_{m, n},
$$

where the vector $\mathcal{E}_{m, n}$ is the FD disturbance. The SFP performs CCI suppression on $\mathcal{Y}_{n}$ using the FD vector $\mathcal{W}_{m, n}$ to produce the output

$$
\begin{aligned}
\mathcal{Z}_{m} & =\sum_{n=1}^{N} \mathcal{W}_{m, n} \circ\left[\boldsymbol{H}_{m, n} \circ \mathcal{D}_{m}+\mathcal{E}_{m, n}\right] \\
& =\mathcal{D}_{m} \circ \sum_{n=1}^{N} \mathcal{W}_{m, n} \circ \mathcal{H}_{m, n}+\sum_{n=1}^{N} \mathcal{W}_{m, n} \circ \mathcal{E}_{m, n}
\end{aligned}
$$

We can write this in terms of the effective channel frequency response vector

$$
\mathcal{F}_{m}=\sum_{n=1}^{N} \mathcal{W}_{m, n} \circ \mathcal{H}_{m, n},
$$

and the residual interference vector

$$
\mathcal{I}_{m}=\sum_{n=1}^{N} \mathcal{W}_{m, n} \circ \mathcal{E}_{m, n} .
$$

Therefore, (27) becomes

$$
\mathcal{Z}_{m}=\mathcal{D}_{m} \circ \mathcal{F}_{m}+\mathcal{I}_{m}, \quad m \in[1 \ldots M] .
$$

An MMSE estimate of $\mathcal{D}_{m}$ that is linear in $\mathcal{Z}_{m}$ can now be computed as

$$
\hat{\mathcal{D}_{m}}=\mathcal{G}_{m} \circ \mathcal{Z}_{m},
$$

where $\mathcal{G}_{m}$ is the MMSE FDE for the $m^{t h}$ data stream. It minimizes the mean squared error (MSE) between $\hat{\mathcal{D}_{m}}$ and $\mathcal{D}_{m}$,

$$
\mathrm{MSE}=E\left[\left(\left\|\hat{\mathcal{D}_{m}}-\mathcal{D}_{m}\right\|\right)^{2}\right]=E\left[\left\|\mathcal{G}_{m} \circ \mathcal{Z}_{m}-\mathcal{D}_{m}\right\|^{2}\right] .
$$

Substituting (30) into this we obtain

$$
\mathrm{MSE}=E\left[\left\|\mathcal{G}_{m} \circ\left[\mathcal{D}_{m} \circ \mathcal{F}_{m}+\mathcal{I}_{m}\right]-\mathcal{D}_{m}\right\|^{2}\right] .
$$

Using the triangle inequality the MSE is upper bounded by

$$
\begin{aligned}
\mathrm{MSE} \leq & E\left[\left\|\mathcal{D}_{m} \circ\left(\mathcal{G}_{m} \circ \mathcal{F}_{m}-\mathbf{1}\right)\right\|^{2}\right. \\
& +2\left\|\mathcal{D}_{m} \circ\left(\mathcal{G}_{m} \circ \mathcal{F}_{m}-\mathbf{1}\right)\right\|\left\|\mathcal{G}_{m} \circ \mathcal{I}_{m}\right\| \\
& \left.+\left\|\mathcal{G}_{m} \circ \mathcal{I}_{m}\right\|^{2}\right],
\end{aligned}
$$

where $\mathbf{1}=[1,1, \ldots, 1]$ is a vector of ones. We assume that the data samples are statistically independent, have zero mean and variance equal to $\rho_{d}^{2}$. We also assume that the resulting $\mathcal{I}_{m}$ contains negligible interference due to SFP and hence is white with variance $\rho_{i}^{2}$. Therefore,

$$
\mathrm{MSE} \leq \rho_{d}^{2}\left(\left\|\mathcal{G}_{m} \circ \mathcal{F}_{m}-\mathbf{1}\right\|^{2}\right)+\rho_{i}^{2}\left\|\mathcal{G}_{m}\right\|^{2} .
$$

Taking the partial derivative of this with respect to the $j^{t h}$ element of the equalizer vector $\mathcal{G}_{m}$ for $j=\left[1,2, \ldots, N_{s}\right]$ and setting the resulting system of equations to zero we have the optimal MMSE FDE in the $m^{\text {th }}$ branch as the vector

$$
\mathcal{G}_{m}=\mathcal{F}_{m}^{*} \circ\left(\mathcal{F}_{m} \circ \mathcal{F}_{m}^{*}+\frac{\rho_{i}^{2}}{\rho_{d}^{2}} \mathbf{1}\right)^{-1} .
$$

\section{SIMULATION RESULTS}

In the simulations, QPSK is used in a four-transmit, fourreceive antenna system. Each transmission frame of length $N_{s}=1024$ contains 128 training symbols. Root-raised cosine transmit and receive pulse shaping with a roll-off of 0.4 is used. A three ray sparse multipath channel with a maximum ISI span of 20 symbol periods is considered. We assume that the channel remains static over a transmission frame and that the complex channel taps have zero-mean and unit variance. The signal to noise ratio (SNR) is defined as the average received signal energy per bit from all transmitters to the noise per receive antenna. The bit error rate (BER) is obtained by averaging the instantaneous BER over many transmission frames.

Fig 3 illustrates the effect on the performance of the number of degrees of freedom $L$ of the FIR filter, $\mathbf{w}_{m, n}$. A SFE computed with $L \geq(v+1)$ results in the best performance. A 
fundamental issue in practical systems is the required length of the FIR filter to handle a given ISI span. The larger the ISI span the larger the required length of $\mathbf{w}_{m, n}$ and the larger the complexity. Given a maximum ISI span of $v$, the integrated SFE with $L \geq(v+1)$ outperforms 3-stages of the LSF scheme of [1] and is only $1.2 \mathrm{~dB}$ poorer than 4-stages at a BER of $10^{-3}$. The complexity of the integrated structure of SFE can be shown to be lower than the overall complexity of the LSF of [1] with 4-stages. In addition, the processing delay due to the parallel structure is lower than the LSF structure of [1] which implements SIC in the FD.

Fig 4 illustrates that similar performance is achieved for all transmitted streams. This is an important advantage of the proposed integrated SFE receiver over [1].

The effect of the number of spatial degrees of freedom or the spatial receive diversity effect on the performance of the integrated SFE is also shown in Fig 4. At a BER of $10^{-3}$ the system using three receivers performs 8 to $9 \mathrm{~dB}$ worse than the one with an equal number of transmitters and receivers. An additional receiver, $N=5$, improves the performance due to the increased number of spatial degrees of freedom and improved receive diversity.

\section{CONCLUSIONS}

We have proposed an integrated SC-SFE receiver architecture for combined CCI suppression and ISI equalization. Simulation results show that the proposed receiver achieves excellent performance even for channels with large ISI spans. Due to the parallel structure of the SFE, the overall complexity is lower than that of interference cancellation based LSF approaches such as [1], [9], [8], [7]. In addition, in the proposed SC-SFE system all data streams yield similar performance.

\section{REFERENCES}

[1] X. Zhu and R. D. Murch, "Layered space-frequency equalization in a single carrier MIMO system for frequency selective channels," IEEE Trans. Wireless Commun., vol. 3, no. 3, pp. 701-708, May 2004.

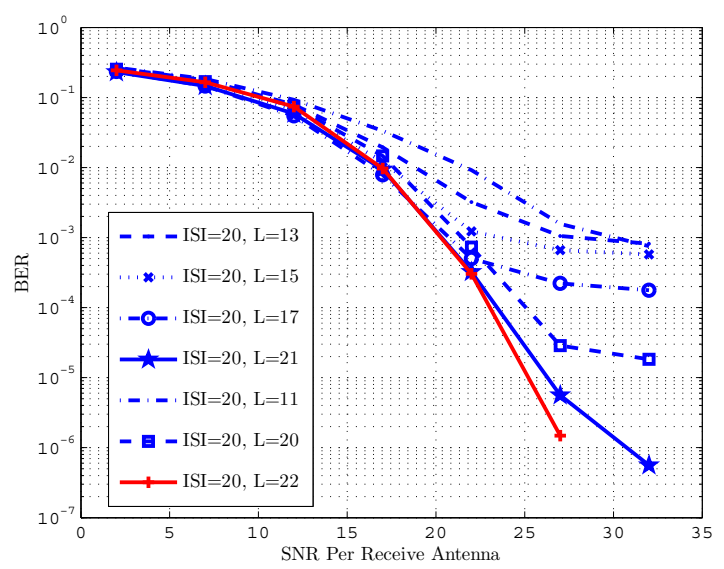

Fig. 3. Effect of FIR filter length $L$ on the overall performance for $M=$ $N=4$.

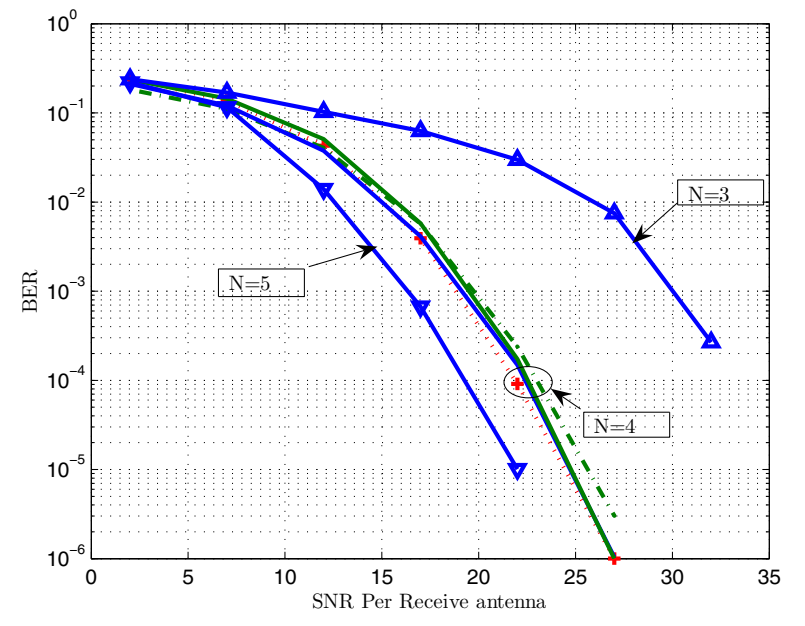

Fig. 4. Performance of integrated SFE for $N=3,4,5$ receive antennas. The performance of all transmitted data streams is shown for $M=N=4$.

[2] W. van Etten, "Maximum likelihood receiver for multiple channel transmission systems," IEEE Trans. Commun., vol. 24, no. 2, pp. $276-$ 283, Feb. 1976.

[3] D. Falconer, S. Ariyavisitakul, A. Benyamin-Seeyar, and B. Eldson, "Frequency domain equalization for single-carrier broadband wireless systems," in IEEE Commun. Mag., Apr. 2002, pp. 58-66.

[4] J. Coon, J. Siew, M. Beach, A. Nix, S. Armour, and J. McGeehan, "A comparison of MIMO-OFDM and MIMO-SCFDE in WLAN environments," in Proc. IEEE Globecom, vol. 6, Dec. 2003, pp. 32963301.

[5] R. Kalbasi, R. Dinis, and D. Falconer, "Hybrid time-frequency layered space-time receivers for severe time dispersive channels," in Proc. SPAWC, Portugal, Lisbon, July 2004, pp. 218-222.

[6] P. Du, G. Bi, and Q. Li, "Single carrier frequency domain equalization for high data rate transmission systems over frequency selective fading channels," in Proc. IEEE 6th CAS Symp. on Emerging Technologies:Mobile and Wireless Comm., May 2004, pp. 361-364.

[7] R. Dinis, R. Kalbasi, D. Falconer, and A. Banihashemi, "Channel estimation for MIMO systems employing single-carrier modulations with iterative frequency-domain equalization," in Proc. VTC, vol. 5, Sept. 2004.

[8] Y. Zhu and K. B. Lataief, "Single-carrier frequency domain equalization with noise prediction for MIMO systems," IEEE Trans. Commun., vol. 55, no. 5, pp. 1063-1076, May 2007.

[9] R. Steffen, B. Tufik, and H. Mario, "Successive interference cancellation for SC FDE MIMO systems," in Proc. PIMRC, vol. 2, Oct 2006, pp. 1142-1146.

[10] J. Siew, J. Coon, R. J. Piechocki, A. Dowler, A. Nix, M. Beach, S. Armour, and J. M. Geehan, "A channel estimation algorithm for MIMO-SCFDE," IEEE Commun. Lett., vol. 8, no. 9, pp. 555-557, Sept. 2004.

[11] E. G. Larsson and P. Stoica, Space-time Block Coding for Wireless communications. Cambridge, UK: University Press, 2003.

[12] L. Guo and Y.-F. Huang, "A multi-user SC-FDE-MIMO system for frequency-selective channels," in Proc. Thirty-Ninth Asilomar Conf. on Signals, Systems, and Computers, Oct. 2005, pp. 1593-1597.

[13] J. Siew, J. Coon, R. Piechocki, A. Nix, M. Beach, S. Armour, and J. M. Geehan, "A bandwidth efficient channel estimation algorithm for MIMO-SCFDE," in Proc. VTC, vol. 2, Oct 2003, pp. 1142-1146.

[14] J. W. Liang and A. J. Paulraj, "Two-stage CCI / ISI reduction with space-time processing in TDMA cellular networks," in Proceedings of 30th Asilomer Conf. on Signals, Sytems and Computers, Vol. 1, Oct. 1996, pp. 607-611. 